\title{
The Nordic Tools for advanced analysis of interferometry data
}

\author{
Ivan Martí-Vidal, Wouter H. T. Vlemmings, Lukas Lindroos, Sebastién Muller \\ Dpt. of Earth and Space Sciences, Chalmers University of Technology \\ Onsala Space Observatory, SE-43992, Onsala (Sweden) E-mail: mivan@ chalmers . se
}

The Nordic tools for Interferometry are a set of algorithms developed by the Nordic Node of the ALMA Regional Center (Nordic ARC) and its collaborators. These are tools specially designed for an advanced processing and analysis of astronomical interferometric observations. Here we present a subset of our tools and also give a list of tools currently being implemented. Please visit our website for more information:

http://nordic-alma.se/support/software-tools

Any comment, suggestion, and/or bug report can be sent to contact@nordic-alma.se

12th European VLBI Network Symposium and Users Meeting,

7-10 October 2014

Cagliari, Italy 


\title{
The Nordic tools for Interferometry
}

\author{
I. Martí-Vidal, W. Vlemmings, L. Lindroos, S. Muller, et al. \\ Onsala Space Observatory \\ Chalmers Univ. of Technology (Sweden)
}

The Nordic tools for Interferometry are a set of algorithms developed by the Nordic Node of the ALMA Regional Center (ARC) and its collaborators. These are tools specially designed for an advanced processing and analysis of astronomical interferometric observations. Here we

present a subset of our tools and also give a list of tools currently being implemented.

Please visit our website for more information: http://nordic-alma.se/support/software-tools

Any comment, suggestion, and/or bug report can be sent to contact@nordic-alma.se

UVMULTIFIT is a versatile tool for fitting generic source models to the visibilities. Any combination of model components, with a generic frequency dependence in any of their defining parameters, can be specified. Any algebraic relationship among the defining parameters of the model components can also be specified (see examples below). Fits to continuum and/or spectral lines are supported. Limited support also for mosaic observations.

See Marti-Vidal et al. 2014, A\&A, 563, A136

(Also IMMULTIFIT, an extension working in the image domain).

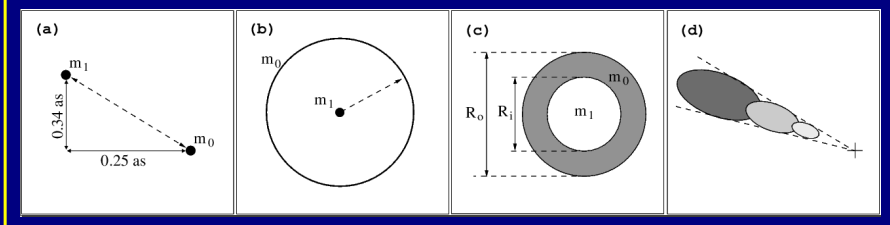

EXAMPLE SOURCES: (a) two points with fixed relative position, but free absolute position. (b) a disc
plus a point at its center (the absolute position of the two sources and/or the disc size and intensity plus a point at its center (the absolute position of the two sources and/or the disc size and intensity
can also be set free). (c) a disc with a hole (built as another disc with negative intensity). (d) an can also be set free). (c) a disc with a hole (built as another disc with negative intensity). (d) an
optically-thick jet (i.e., with a core shift). In all these cases, the free model components can depend optically-thick jet (i.e., with a core shift). In all these cases, the free
on any algebraic function of frequency and/or fitting parameters.

PRACTICAL (REAL) EXAMPLE: ALMA OBSERVATIONS OF THE LENSED BLAZAR PKS1830-211 AT $93 \mathrm{GHz}$

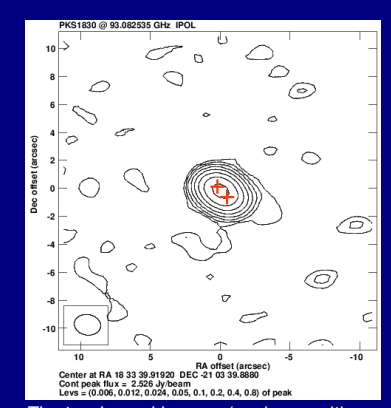

The two lensed images (each one with a

different absorption-line spectrum) are fully

blended in the image plane, due to the

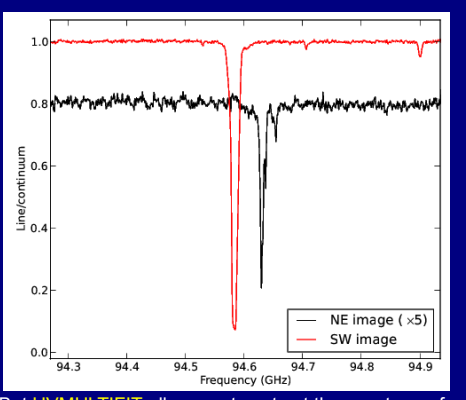

But UVMULTIFIT allows us to extract the spectrum of each image without blending, by fitting two sources with a known separation (but free absolute position i.e., example (a) in the figure above.

OTHER TOOLS (DEVELOPED OR BEING DEVELOPED): FAKEOBS: Allows the user to substitute data from real observations with model visibilities, obtained from Fourier inversion of a FITS image (cube). This is useful for simulations, proposal preparation, tests, and even for data modelling (a rough alternative to UVMULTIFIT, if the user computes the Chi Square minimization by him/herself).

CUBE-ANIMATE: A multimedia tool to make movies from data cubes.

UV-STACKING, developed by L. Lindroos, is a too to perform stacking of weak sources in the Fourier domain. This approach has many advantages, compared to the image-based stacking.

See Lindroos et al. MNRAS (submitted)
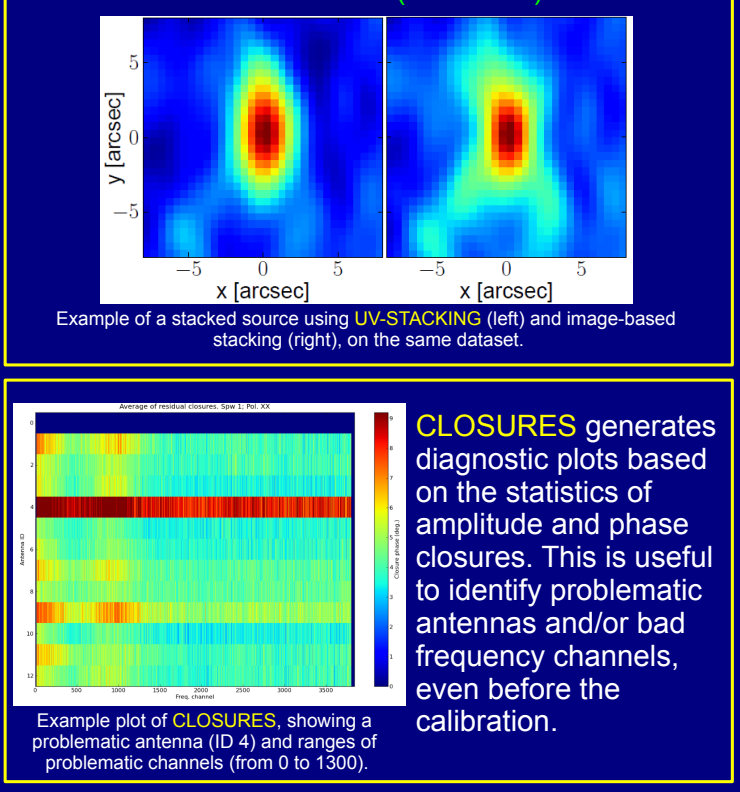

CHANDRAFERMI, developed in collaboration with the German ARC Node (Bonn), allows the user to estimate magnetic fields from full-polarization images, using the Chandrasekhar-Fermi algorithm. Chandrasekhar, S., Fermi., E. 1953, ApJ 118, 113

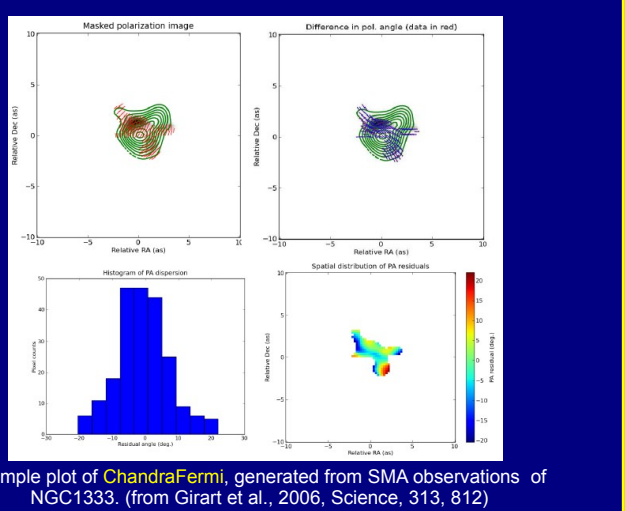

